\title{
On Packing Thirteen Points in an Equilateral Triangle
}

\author{
Natalie Tedeschi* \\ Department of Mathematical Sciences, Carnegie Mellon University, Pittsburgh, PA \\ https://doi.org/10.33697/ajur.2021.042 \\ Student: ntedesch@andrew.cmu.edu \\ Mentor:jmackey@andrew.cmu.edu
}

\begin{abstract}
The conversation of how to maximize the minimum distance between points - or, equivalently, pack congruent circles - in an equilateral triangle began by Oler in the 1960s. In a 1993 paper, Melissen proved the optimal placements of 4 through 12 points in an equilateral triangle using only partitions and direct applications of Dirichlet's pigeon-hole principle. In the same paper, he proposed his conjectured optimal arrangements for 13, 14, 17, and 19 points in an equilateral triangle. In 1997, Payan proved Melissen's conjecture for the arrangement of fourteen points; and, in September 2020, Joos proved Melissen's conjecture for the optimal arrangement of thirteen points. These proofs completed the optimal arrangements of up to and including fifteen points in an equilateral triangle. Unlike Melissen's proofs, however, Joos's proof for the optimal arrangement of thirteen points in an equilateral triangle requires continuous functions and calculus. I propose that it is possible to continue Melissen's line of reasoning, and complete an entirely discrete proof of Joos's Theorem for the optimal arrangement of thirteen points in an equilateral triangle. In this paper, we make progress towards such a proof. We prove discretely that if either of two points is fixed, Joos's Theorem optimally places the remaining twelve.
\end{abstract}

\section{KEYWORDS}

optimization; packing; equilateral triangle; distance; circles; points; thirteen; maximize

\section{INTRODUCTION}

Packing is a class of optimization problems. The objective is to place some number of non-overlapping geometric objects such that they are entirely contained in a larger object leaving as little space remaining in the larger object as possible. Packing problems have an expansive history beginning from when Kepler conjectured the density of the densest ball packing in $1611 .{ }^{1}$ In this paper, we consider the problem of packing thirteen congruent circles in an equilateral triangle. It is important to note here the relationship between the problem of packing thirteen congruent circles into an equilateral triangle and maximizing the minimum distance between thirteen points in an equilateral triangle. As Figure 1 below shows, the centers of the circles in the optimal packing of thirteen congruent circles in the larger triangle are the points that maximize the minimum distance between thirteen points in the smaller triangle. Thus, these are the same problem.

The two interpretations of the problem lead to different applications. By looking at the problem as a packing of circles, we answer questions along the lines of how large a box needs to be in order to hold $n$ bottles of water. By considering the problem in terms of maximizing the distance between points on a plane, we solve problems like how to optimally place transistors onto a microchip. Throughout this paper, we will consider the problem in terms of maximizing the minimum distance between thirteen points in an equilateral triangle. 


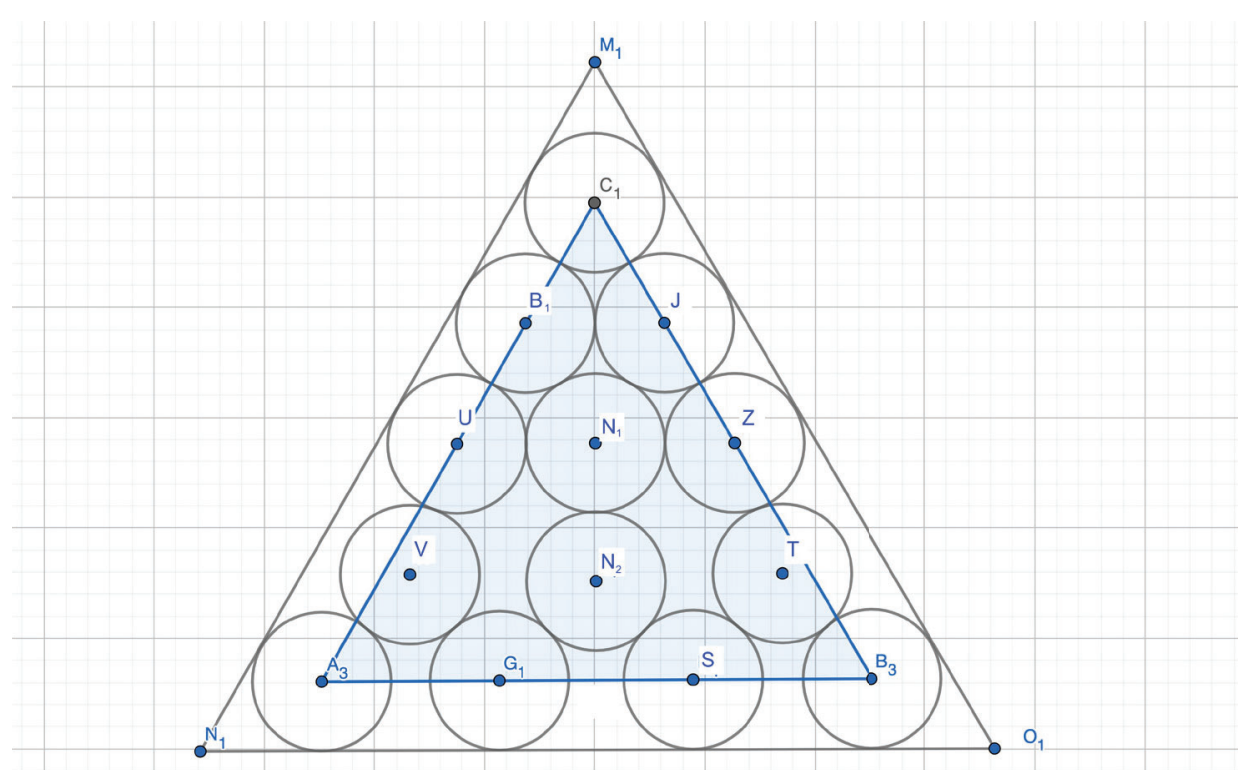

Figure 1. An equilateral triangle with optimal point packing within an equilateral triangle with optimal circle packing

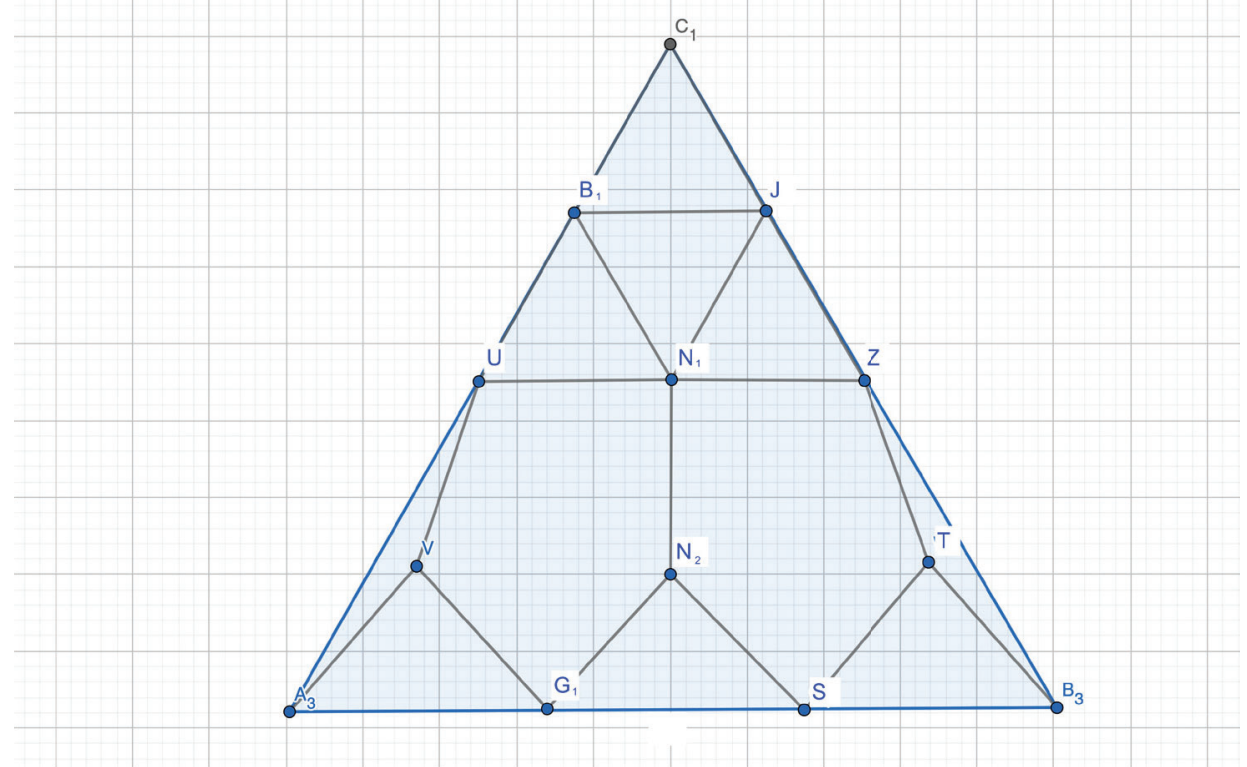

Figure 2. Optimal configuration of thirteen points in an equilateral triangle

Configurations that maximize the minimum distance between $n$ points in an equilateral triangle have been proven for $n \leq 12,{ }^{2,3} n=14,20,{ }^{4} n=k(k+1) / 2$ for any $k \in N,{ }^{5}$ and now $n=13 .{ }^{6} k(k+1) / 2$ is the $k^{\text {th }}$ triangular number, and the optimal packing is the obvious one: in rows of $1,2,3, \ldots, k$ points. The proof for $n=13$ was completed by Joos in 2020, confirming Melissen's 1993 conjecture that the orientation of thirteen points in an equilateral triangle shown in Figure 2 uniquely maximizes the minimum of the distance between two points. ${ }^{2}$ In other words, no other configuration of thirteen points in an equilateral triangle exists such that the distance between every pair of points is more than or equal to the minimum distance between the points in Figure 2. We will denote this minimum distance as $d_{13}$.

Joos's proof for the optimal configuration of thirteen points in an equilateral triangle diverged from the strategies that had been used previously to find optimal configurations of points in equilateral triangles. Rather than using only discrete mathematics, his proof requires continuous functions and calculus, relies heavily on inequalities, and considers several cases. This paper explores the possibility of an entirely discrete proof of the optimal arrangement of thirteen 
points in an equilateral triangle. We demonstrate that if one can prove that an optimal placement of thirteen points must include $N_{1}$ or $N_{2}$ from Figure 2 then we would have an alternate and discrete proof that the Melissen placement of points its optimal. Determining whether the methods that had worked for proving the optimal arrangements of fewer points are still valid for thirteen points could influence whether people must search for new approaches as the number of points continues to increase.

Joos computes $d_{13}=9-5 \sqrt{3}-\frac{7 \sqrt{6}}{2}+6 \sqrt{2} \approx 0.251813$ for a triangle with side length $1 .^{6}$ Pairs of points in Figure 2 above whose distance is $d_{13}$ are connected by a grey line.

\section{RESULTS}

We know from Melissen's 1993 paper that the configuration of points shown in Figure 2 exists such that pairs of points connected by a grey line in the figure are $d_{13}$ apart. $^{2}$ From there, his conjecture states that this configuration is both optimal and unique, meaning that there is no other configuration of thirteen points in an equilateral triangle such that every pair of points is more than or equal to $d_{13}$ apart. We will prove two theorems: one showing that if we assume the position of $N_{1}$ from Figure 2, then Joos's Theorem for thirteen points holds; and the other showing that if we assume the position of $N_{2}$, then Joos's Theorem holds as well.

We first note that Melissen proved the following lemma:

Lemma. In an optimal configuration of $n \geq 3$ points in an equilateral triangle, the three vertices of the triangle must be among the selected points. ${ }^{3}$

Theorem 1. Let $N_{1}$ from Figure 2 be the point that is $d_{13}$ from each of the points on adjacent sides of the triangle that are $d_{13}$ from the top corner. If $N_{1}$ is fixed, then Joos's Theorem for the optimal arrangement of 13 points is correct.

In other words, given the position of $N_{1}$, there is no arrangement of the remaining twelve points in the triangle other than the arrangement shown in Figure 2 such that every pair of two of the thirteen points is at least $d_{13}$ apart. We first realize that for such a configuration to exist, it cannot contain any other points in the interior of a circle with radius $d_{13}$ centered at $N_{1}$. As Figure 3 shows, this creates two distinct regions: the region above the circle about $N_{1}$ and the region below. We will begin by examining the upper region.

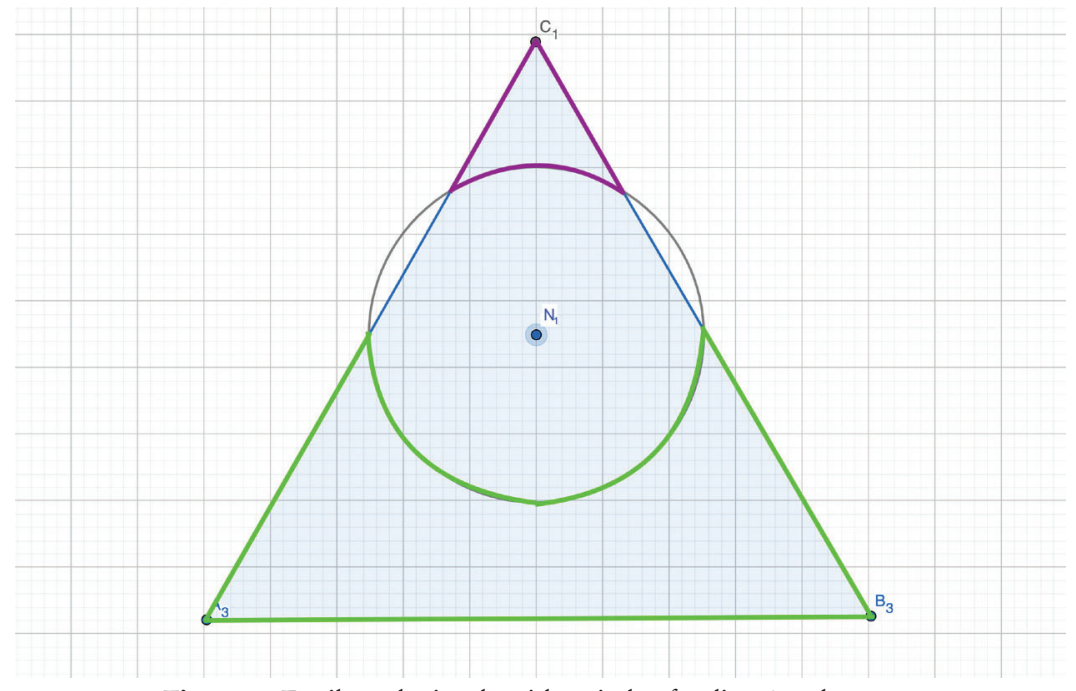

Figure 3. Equilateral triangle with a circle of radius $d_{13}$ about $N_{1}$

Given our definition of $N_{1}$ and as Figure 4 illustrates, the intersection of the interiors of circles of radius $d_{13}$ about each of the three corners of the upper region contain every point except the three corners. Thus we know that every point other than the corners is less than $d_{13}$ every other point in the region. So in order for there to be at least two 
points in this region whose distance apart is at least $d_{13}$, those points must be the corners. This also shows that we can include at most three points in this region. We can achieve this only by placing them in the corners, so the arrangement of three points in this region is unique. Since we can fit at most three points in this region, we must put at least nine points in the lower region.

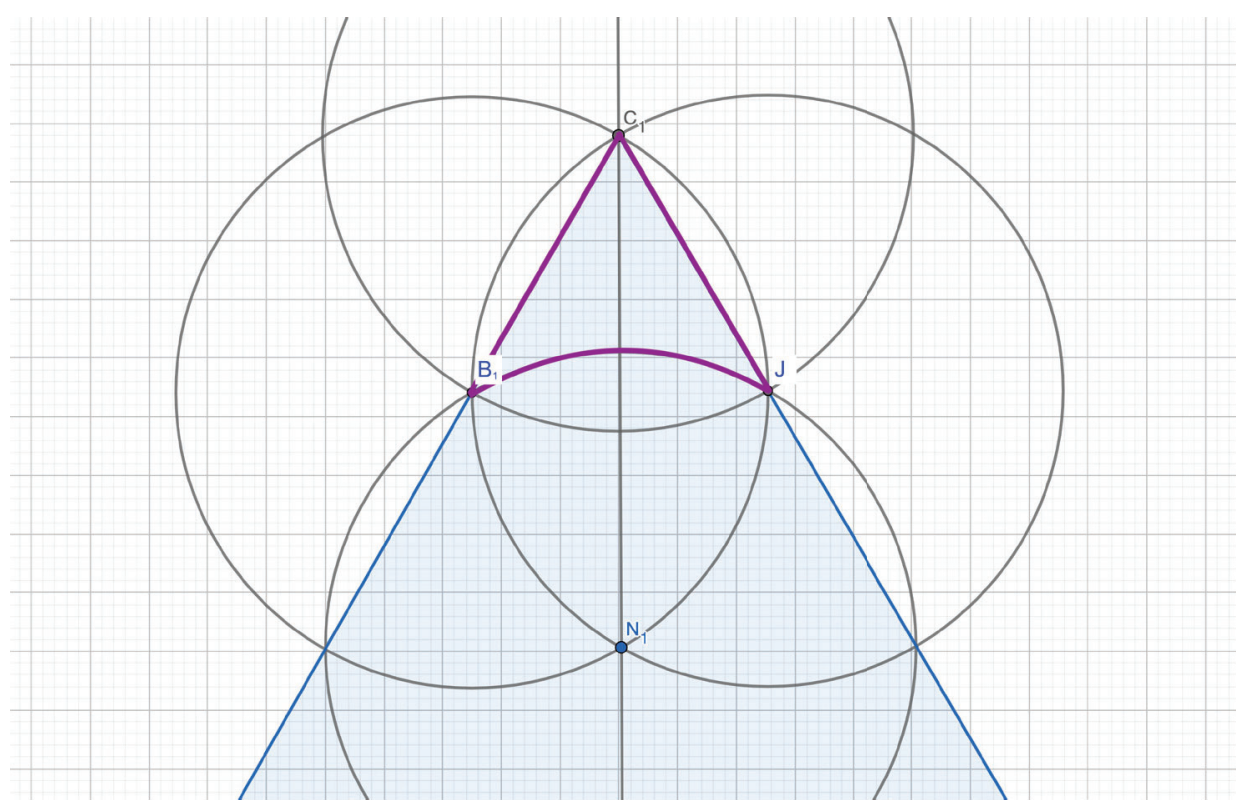

Figure 4. Equilateral triangle with upper region outlined

The decomposition shown in Figure 5 partitions the lower region into eight subregions: the interiors of circles with radius $d_{13}$ about $U$ and $Z$ (the highest points in the lower region), the interiors of the circles with radius $d_{13}$ about the bottom corners, the interiors of the circles with radius $d_{13}$ about $V$ and $T$ (the intersection points for the first two circles) minus the first two circles, and then the remaining space split symmetrically down the center. The lower region includes its upper boundary, so each of the five arcs that make up this boundary are included in their respective subregions. The purple and orange subregions include their boundaries except where they intersect with brown subregions; these intersections belong to the brown subregions. The brown subregions include their boundaries except where they intersect with the blue subregions. The blue subregions include their entire boundaries.

Since there are nine points, without loss of generality, we must place five left of and including the center. There are five points to place in four subregions. Thus, at least one subregion must have at least two points. Since the circular regions do not include their entire boundaries, they cannot fit more than one point. We take a closer look at the region outlined in blue from Figure 5 in Figure 6 below.

Figure 6 shows the region outlined in blue along with a circle of radius $d_{13}$ about each of its corners. The circles about the corners of the region - $N_{2}, H_{1}, G_{1}$, and $M_{1}$ - are green, pink, orange, and brown respectively. Since the pink and brown circles contain the entire region, it is clear that any point in the blue region is less than $d_{13}$ from $H_{1}$ and $M_{1}$. From Melissen's orginal paper, we know that $N_{2}$ (the point $d_{13}$ from and directly below $N_{1}$ ) and $G_{1}$ (the point $d_{13}$ from $V$ on the side of the triangle) are $d_{13}$ apart. $^{2}$ Thus, the only way to fit two points in this region is at $N_{2}$ and $G_{1}$.

We have established that the only subregion from Figure 5 that can fit two points is the blue region and we cannot fit three points in any subregion. So we must put two points in the blue subregion at $N_{2}$ and $G_{1}$ and one point in each of the other subregions. We know from our lemma that there must be a point at $A_{3}$ (the corner). Now the only point 


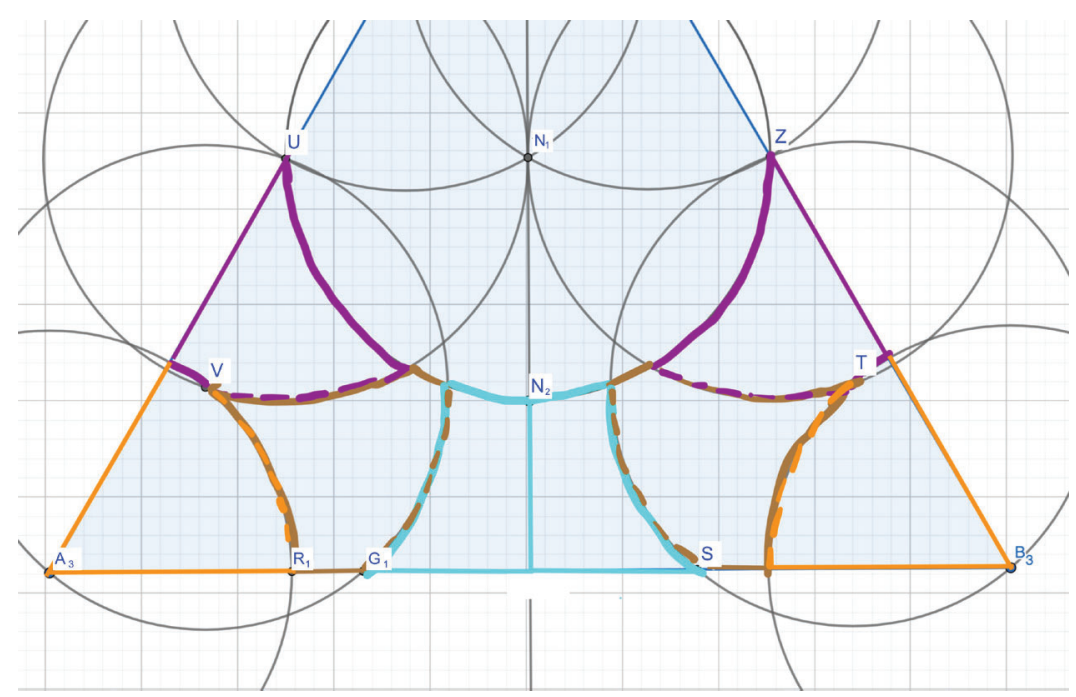

Figure 5. Decomposition of lower region of equilateral triangle

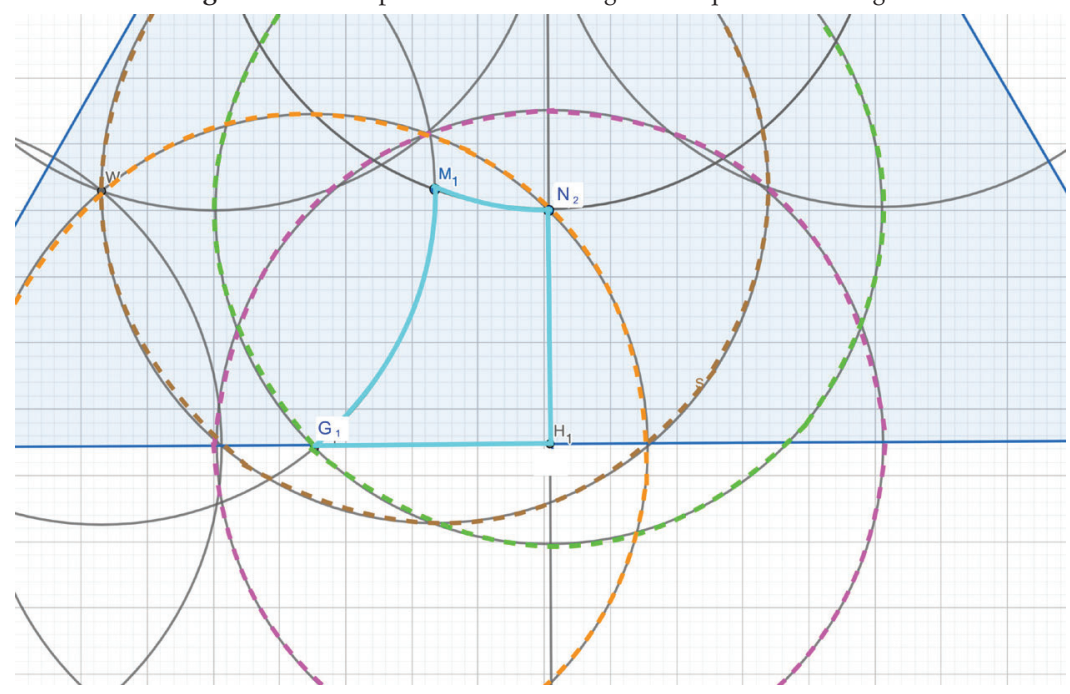

Figure 6. Blue subregion with circles about each corner

in the brown subregion that is $d_{13}$ from $G_{1}$ and $A_{3}$ is $V$ so we must place the next point there. The only point in the purple region that is $d_{13}$ from $V$ is $U$, so a point belongs there as well. By the same argument, the points on the right side must be placed in the corresponding locations. We have shown that we can fit at most three points in the upper region and nine points in the lower region and that the arrangements of points in both of those regions is unique, so we have uniquely placed all twelve points. This concludes Theorem 1.

Theorem 2. Let $N_{2}$ from Figure 2 be the point on the altitude from $C_{1}$ that is $d_{13}$ from $N_{1}$. If $N_{2}$ is fixed, then Joos's Theorem for the optimal arrangement of 13 points is correct.

In order to prove Theorem 2, we will first split the triangle into an upper and lower region and decompose the upper region to show that we can fit at most five points in the upper region. We will then decompose the lower region into identical halves, providing that one of these halves must contain four points. We will then show that an arrangement of four points into one of the halves is unique. Fixing these points will further limit the arrangements of points in the upper region, allowing us to change our decomposition and prove that we can actually only fit four points in the upper region, so therefore, there are four points in the other half of the lower region as well. We will then prove that there is a unique configuration of four points in the remaining half of the lower region and four points in the upper region, thus giving a unique configuration of all of the remaining twelve points. 


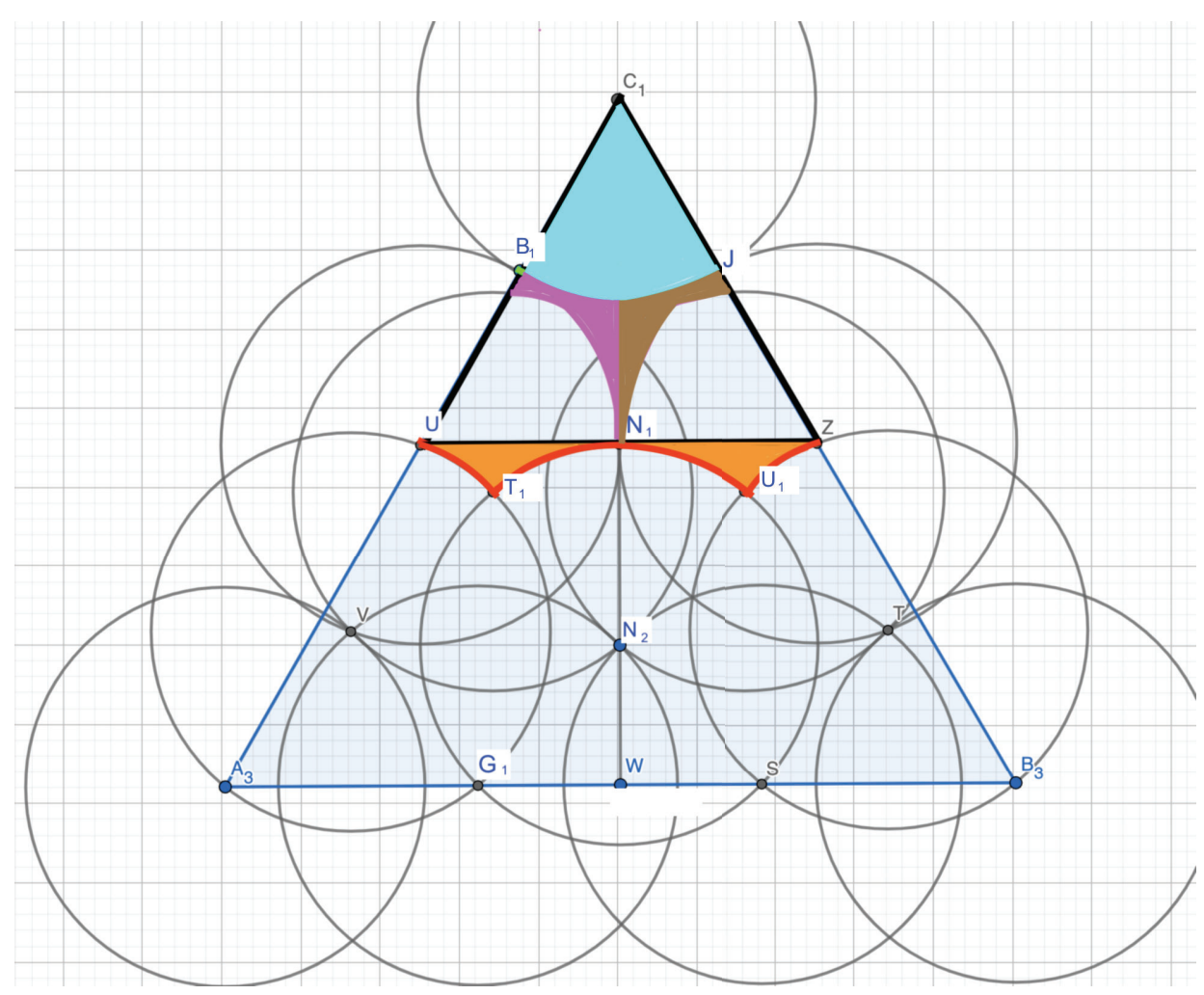

Figure 7. Decomposition of an upper region of an equilateral triangle

In order to maintain at least $d_{13}$ between each point, there cannot be another point within a circle of radius $d_{13}$ centered at $N_{2}$. Figure 7 draws a red curve along the boundary of the union of a circles of radius $d_{13}$ about the points $N_{2}, V$ and $T$ from Figure 2. We will consider the region above and including the red curve - but excluding the points $U$ and $Z$ - as the upper region, and the region below the red curve plus $U$ and $Z$ as the lower region.

Consider equilateral triangle $\triangle C_{1} U Z$ outlined in black in Figure 7. We will first show that there can be at most four points in this triangle excluding $U$ and $Z$. Each side of this triangle has length $2 d_{13}$ since the points are defined such that $B_{1}$ is $d_{13}$ from $C_{1}$ and $U$ is $d_{13}$ from $B_{1}$. By Melissen's proof optimizing six points in an equilateral triangle, we can uniquely fit six points into this triangle such that no pair of points is less than $d_{13}$ apart at $C_{1}, B_{1}, J, U, Z$, and $N_{1}{ }^{2}$ Since this configuration is unique, if we exclude points $U$ and $Z$ from the region, we can no longer fit six points in the triangle outlined in black. Furthermore, since the optimal configuration of five points in an equilateral triangle requires five points from the configuration of the six points labeled in the region, ${ }^{2}$ removing those two points from the region makes it so that we cannot fit five points $d_{13}$ apart from one another either. Thus, in the equilateral triangle outlined in black excluding $U$ and $Z$, we can fit at most four points.

It follows that in order to fit six points in the upper region, we would need to put two points between the black triangle and the red curve shaded in orange - one on each side of $N_{1}$ since we cannot fit two points on either one side given that $N_{1}$ is $d_{13}$ from $U$ and $Z$. We will consider these two orange regions separated by $N_{1}$.

In order for there to be a point in each of these regions, there can be no point in the intersection of the circles about $N_{1}, U$, and $T_{1}$ and the intersection of circles about $N_{1}, U_{1}$, and $Z$ all of radius $d_{13}$, i. e. there can be no points below the pink and brown regions in Figure 7. This is because any point that is in one of those intersections would be less than $d_{13}$ from every point in the corresponding orange region, and we have already established that there must be a point in both regions for there to be six points in the whole upper region. 
We next show that we cannot fit four points in the union of the blue, pink, and brown regions. The blue region is defined as the interior of a circle of radius $d_{13}$ about $C_{1}$, so there cannot be more than one point in this region. The pink and brown regions can each also have at most one point. To understand why, keep in mind, we took $B_{1}, J$, and $N_{1}$ from the optimal placement of six points in black equilateral triangle, so we know that they are $d_{13}$ apart. We cannot have a point at $N_{1}$ because we know that there must be a point in each of the orange regions and $U$ and $Z$ are excluded from those regions, so each of those regions contains a point less than $d_{13}$ from $N_{1}$. Thus, none of the blue, pink, and brown regions can fit more than one point, so we can fit at most three points the union of these three regions. Thus, there are at most five points in the upper region.

We now consider the lower region, which is below the red curve from Figure 7 and includes $U$ and $Z$, but excludes the interior of a circle of radius $d_{13}$ about $N_{2}$. Since we know we can only fit five points in the upper region, we must fit seven points in this region. Without loss of generality, we must fit four point on the left side of this region.

Figure 8 decomposes the left side of this lower region into three subregions. We define the orange subregion as the interior of a circle of radius $d_{13}$ about $U$, the green subregion as the interior of a circle of radius $d_{13}$ about $A_{3}$ and the brown as the remaining space. We know from Melissen's paper that $G_{1}$ and $V$ are $d_{13}$ apart. ${ }^{2}$ Thus, the orange and green subregions can each contain at most one point and the brown subregion can contain at most two points uniquely placed at $G_{1}$ and $V$. Since we need to fit four points into these three subregions, we need to fit at least two points in at least one of these subregions, so we must put points at $G_{1}$ and $V$. The only way to put a point in each of the remaining subregions is by putting them at $A_{3}$ and $U$. Thus, the only possible configuration of four points on the left side of this region are $G_{1}, V, A_{3}$, and $U$.

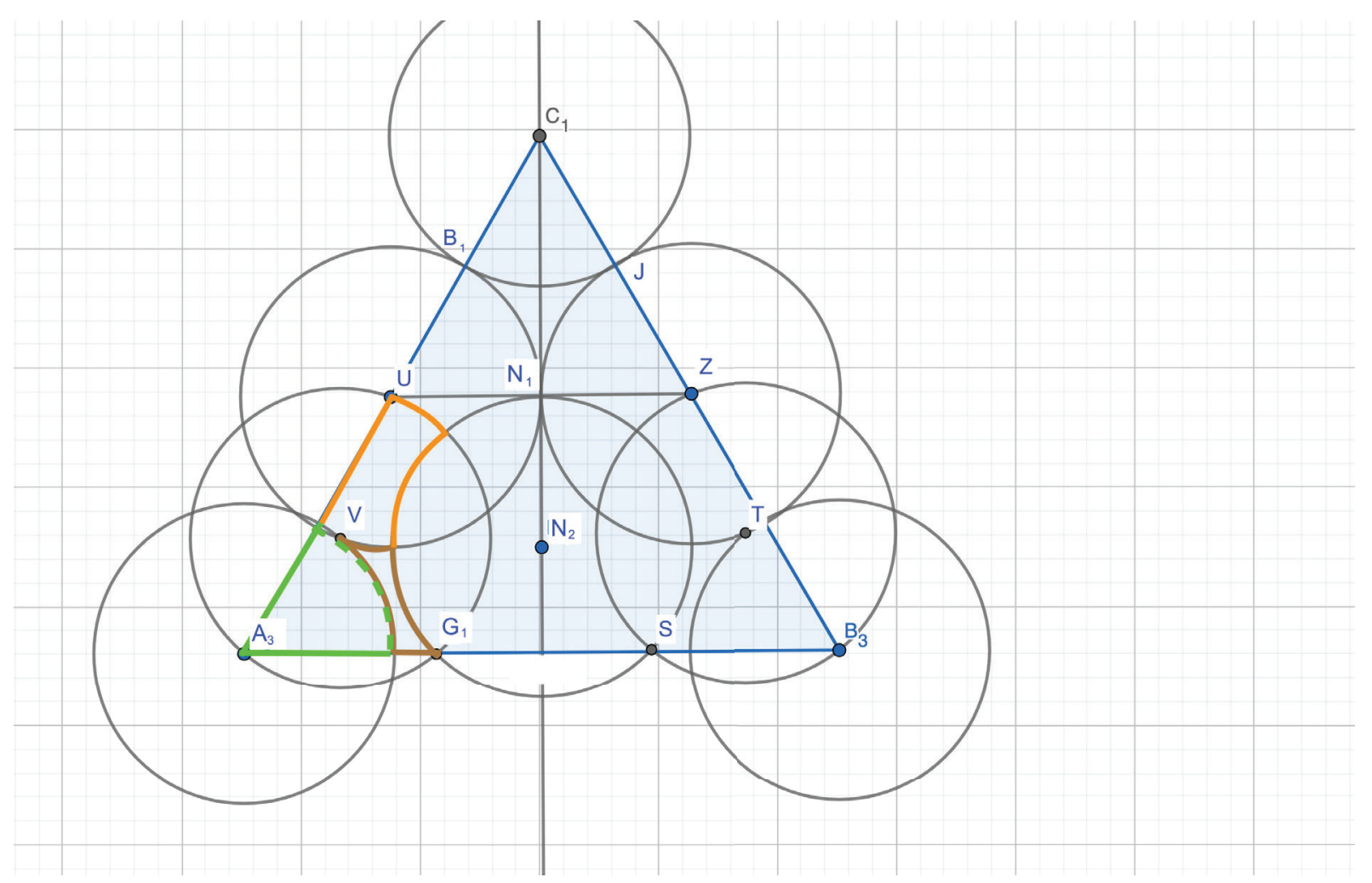

Figure 8. Decomposition of a left lower region of an equilateral triangle

Once we fix those four points, we cannot have any other points within $d_{13}$ of any of them. In particular we cannot have any point in the interior of the circle of radius $d_{13}$ about $U$ which is outlined in pink in Figure 9. With this in mind, Figure 9 revises our decomposition of the upper region using circles of radius $d_{13}$ about $C_{1}$ and $Z$ as boundaries of the regions. Note that $Z$ is not in the upper region at all, $N_{1}$ is in the red region, $J$ is in the yellow region, 
and $B_{1}$ is in the blue region. Since each region can contain at most one point, we can now conclude that there are at most four points in this entire upper region. This means that there must also be four points in the right side of the lower region. An analogous decomposition to that shown in Figure 8 shows that the points on the right side of the lower region must be in analogous locations to those on the left: $B_{3}, S, T$, and $Z$. In particular, there is a point at $Z$, so the points in the red and yellow subregions in Figure 9 must be at $N_{1}$ and $J$ respectively. Fixing those points also requires that the only points that can be placed in each of the other two regions are $B_{1}$ and $C_{1}$. This concludes the unique configuration of the remaining twelve points.

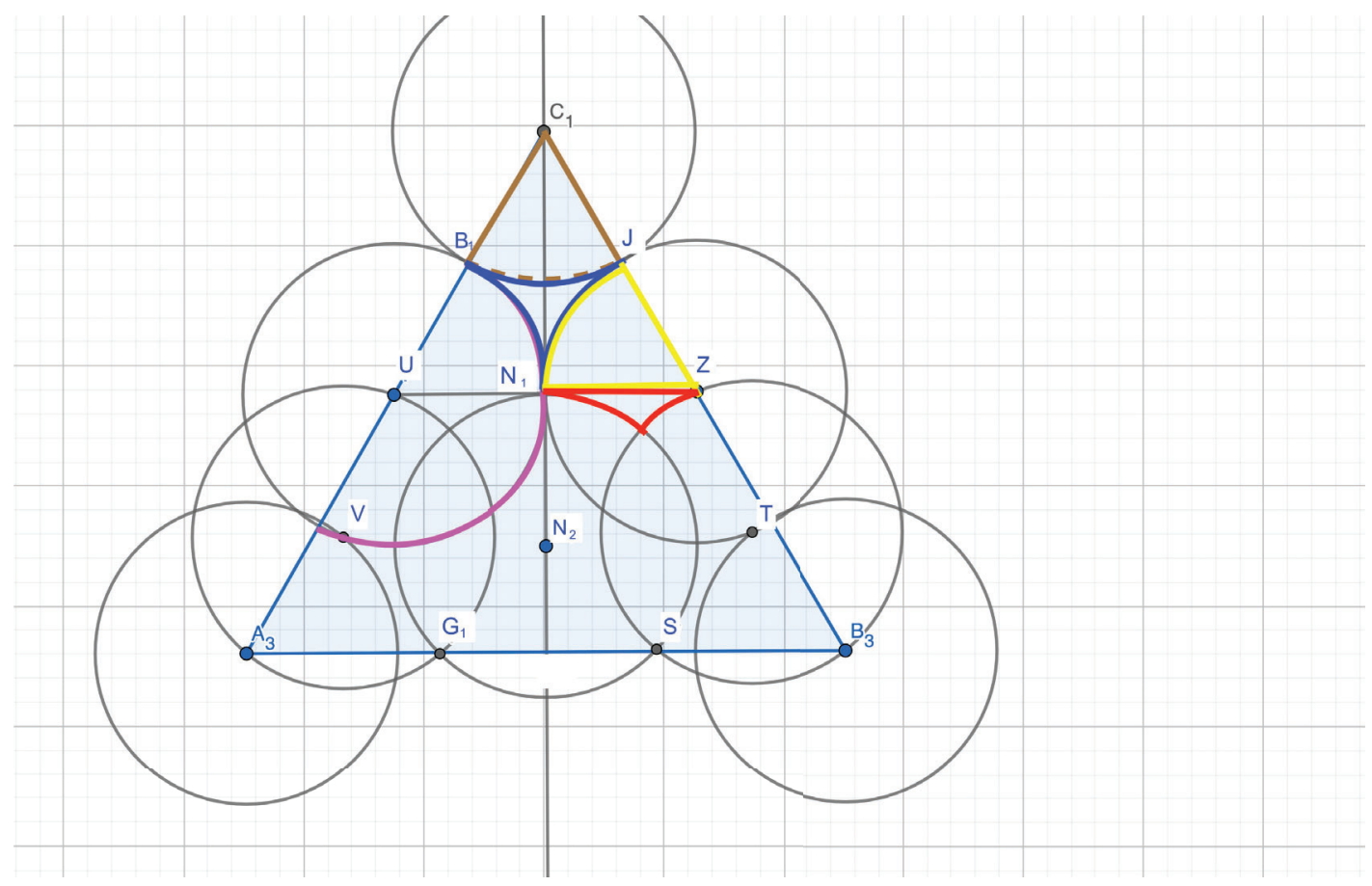

Figure 9. Refined decomposition of the upper region of the equilateral triangle

\section{DISCUSSION}

The proofs provided in this paper bring us closer to a discrete proof of Joos's Theorem for the optimal configuration of thirteen points in an equilateral triangle. We have shown that if either of two points is fixed, then we have an alternate proof of the Melissen configuration of thirteen points. We will now explore how one might begin proving the position of $N_{1}$ or $N_{2}$.

Theorem 3. Recall $N_{1}$ and $N_{2}$ from Figure 2. Let $K, T_{1}$ and $W_{1}, V_{1}$ be in the position that $N_{1}$ and $N_{2}$ would be in if the entire triangle was rotated about the center such that $C_{1}$ was positioned at $B_{3}$ and $A_{3}$ respectively. Let region $H$ be the orange hexagon from Figure 10 defined by $T_{1}, N_{1}, V_{1}, K, N_{2}$, and $W_{1}$ including its boundary. If there are two points in this region at least $d_{13}$ apart, they must be at either $N_{1}$ and $N_{2}, K$ and $T_{1}$, or $W_{1}$ and $V_{1}$ (which by our definition are all the same pair of points up to rotations of the triangle).

Since $H$ is a convex hexagon and congruent across all three altitudes of the large triangle, proving Theorem 3 is equivalent to proving that the distances from $N_{1}$ to $W_{1}, N_{1}$ to $K$, and $W_{1}$ to $K$ are less than $d_{13}$.

The point $E$ in Figure 10 is the midpoint of the segment from $C_{1}$ to $A_{3}$ which is the side length of the triangle. Assuming the triangle has side length 1, the segment from $C_{1}$ to $E$ is 0.5 in length. The segment between $C_{1}$ and $U$ from Figure 2, on the other hand, has length $2 d_{13} \approx 0.503626$. So $E$ from Figure 10 is closer to $B_{1}$ than $U$ from Figure 2 is. Therefore the distance between $E$ and $N_{1}$ is less than $d_{13}$ and equal to the distance between $W_{1}$ and $E$ since $W_{1}$ is the same distance from $A_{3}$ as $N_{1}$ is from $C_{1}$. Thus, $\triangle E N_{1} W_{1}$ is isosceles, so $\angle E N_{1} W_{1} \cong \angle E W_{1} N_{1}$. Since $\triangle C_{1} U Z$ from Figure 2 is equilateral, $\angle C_{1} U N_{1}$ is $60^{\circ}$, so $\angle C_{1} E N_{1}$ in Figure 10 is greater than $60^{\circ}$ as is $\angle A_{3} E W_{1}$. 
Thus, $\angle N_{1} E W_{1}$ is less than $60^{\circ}$, and therefore smaller than $\angle V N_{1} W_{1}$ and $\angle E W_{1} N_{1}$. So, the segment from $W_{1}$ to $N_{1}$ is less than that from $E$ to $N_{1}$ which is less than $d_{13}$. We can generalize this argument to now say that the segments from $N_{1}$ to $W_{1}, N_{1}$ to $K$, and $W_{1}$ to $K$ are less than $d_{13}$ since they are all the same pair of points up to rotations of the triangle. This concludes Theorem 3.

Now in order to show that there are points at $N_{1}$ and $N_{2}$, we need only to show that there must be two points in $H$ because the only way to fit two points in $H$ is by placing points $N_{1}$ and $N_{2}, K$ and $T_{1}$, or $W_{1}$ and $V_{1}$ which are all the same pair of points up to rotations of the triangle.

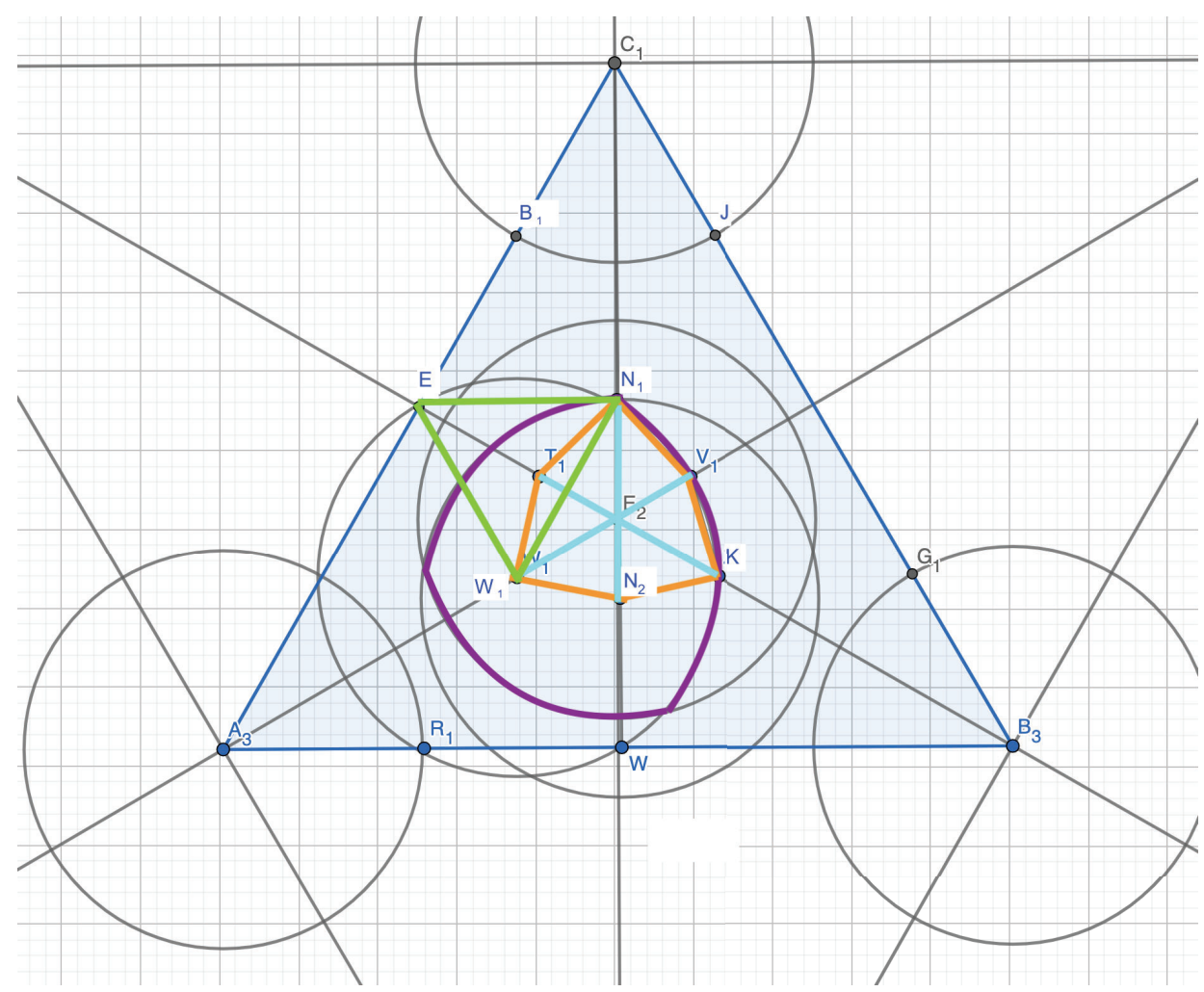

Figure 10. Equilateral triangle with regions of emphasis outlined

In order to show that there are at least two points in $H$, we would first need to show that there must be at least one point in $H$, meaning it is impossible to fit thirteen points outside of $H$ that are at least $d_{13}$ apart. We would then need to show that given that there is a point within $H$, we cannot fit twelve points outside of $H$ that are all at least $d_{13}$ apart. The blue lines clearly partition $H$ into six subregions. Because the six subregions are congruent, once we prove that there is a point in $H$, we can assume without loss of generality that that point is in the bottom left subregion of $H$, the triangle defined by the points $F_{2}, W_{1}, N_{2}$ including the boundary.

If there is a point in that bottom left subregion of $H$, there cannot be another point that is less than $d_{13}$ from all three corners of the subregion or else it would be less than $d_{13}$ from every point in the subregion. Thus, if there is a point in the bottom left subregion of $H$, there cannot be any other point within the intersection of the circles of radius $d_{13}$ about $F_{2}, W_{1}$ and $N_{2}$ which is outlined in purple (excluding the boundary); we will call this region $P$. So in order to complete the discrete proof of Joos's Theorem, all that remains to be proven is that we cannot place thirteen points outside of $H$ and that we cannot fit twelve points outside of the union of $H$ and $P$. 


\section{CONCLUSIONS}

This paper makes progress towards a discrete proof for the optimal orientation of thirteen points in an equilateral triangle by proving that if either of two points is fixed, the other twelve are positioned in accordance with Joos's Theorem. Finding that such a proof exists could influence how mathematicians approach similar packing problems. It is also interesting to note that 13 , along with 8 and 19 can be expressed as $\frac{k(k+1)}{2}-2$ or $\frac{k(k+1)}{2}+2 k+1$ depending on the value of $k$. The first representation shows us that these values are two less than triangular numbers (which are expressed as $\left.\frac{k(k+1)}{2}\right)$, but the second makes sense of the arrangement of the points. Melissen's proof for the optimal configuration of $n=8$ points, and in his conjectures for $n=13$ and $n=19$, all have very similar arrangements. They all have an upper region where the points are arranged as an equilateral triangle with a triangular number of points (that corresponds with the first term of the expression). A discrete proof for $n=13$ could make leeway for a general proof for all $n$ that are two less than a triangular number.

\section{ACKNOWLEDGEMENTS}

The author thanks Professor John Mackey of Carnegie Mellon University for discussions of the problem and reviewing drafts of this manuscript.

\section{REFERENCES}

1. Zong, C. (2014) Packing, covering and tiling in two-dimensional spaces, Expo. Math. 32, 297-364. https://doi.org/10.1016/j.exmath.2013.12.002

2. Melissen, J.B.M. (1993) Densest packings of congruent circles in an equilateral triangle, Amer. Math. Monthly 100, 916-925. https://doi.org/10.2307/2324212

3. Melissen, J.B.M. (1994) Optimal packings of eleven equal circles in an equilateral triangle, Acta Math. Hungar 65, 389-393. https://doi.org/10.1007/BF01876040

4. Payan, C. (1997) Empilement de cercles egaux dans un triangle equilateral. A propos d'une conjecture d'Erdos-Oler, Discrete Math 165-166, 555-565. https://doi.org/10.1016/S0012-365X(96)00201-4

5. Oler, N. (1961) A finite packing problem, Canad. Math. Bull. 4, 153-155. https://doi.org/10.4153/CMB-1961-018-7

6. Joos, A. (2020) Packing 13 circles in an equilateral triangle, Aequat. Math. 35-65.

https://doi.org/10.1007/s00010-020-00753-y

\section{ABOUT THE STUDENT AUTHOR}

Natalie Tedeschi is currently working towards a BS in Discrete Mathematics and Logic at Carnegie Mellon University. She has worked as a teaching assistant in the Department of Computer Science at Carnegie Mellon and as a software engineering intern at Explo, a YCombinator-backed startup. She plans on graduating in May of 2023.

\section{PRESS SUMMARY}

How large is the smallest computer chip that contains $n$ transistors that must be some distance apart from one another? How big must a crate be in order to hold $n$ jugs of water? These questions ask how we can maximize the benefit of costly or harmful materials and provide insight on how we can drive technology and innovation forward. Mathematically, these and many more questions are the same. This paper furthers the discussion of solving these types of problems. 